\title{
ENT trainee papers presented at the Association of Otolaryngologists in Training Conference, 7-8 July 2016, Glasgow, Scotland, UK
}

\author{
President: Mr Robert Nash, North Thames (ENT) \\ Secretary: Ms Natasha Amiraraghi, West of Scotland (ENT) \\ Treasurer: Ms Ruhuta Roplekar, East of Scotland (ENT) \\ Invited panel: Ms May Yaneza, NHS Lanarkshire; Mr Shayan Ansari, NHS Ayrshire and Arran; Mr Charlie Huins, University \\ Hospitals Birmingham NHS Foundation Trust; Prof Anthony Narula, ENT-UK President; Mr Haytham Kubba, NHS Greater \\ Glasgow and Clyde; Mr Craig Murray, NHS Greater Glasgow and Clyde; Mr Angus Cain, NHS Highland; Dr Gillian McDougall, \\ NHS Lothian
}

Junior Otolaryngologist in Training prize (presented by Mr Robert Nash) was awarded to Priya Sethukumar for 'Mind over matter: scar satisfaction and body image in thyroidectomy patients - a prospective study'.

Neck lump clinics: do we still need them?

S Chan, N Amiraraghi, O J Hilmi

Crosshouse Hospital, Glasgow, Scotland, UK

\section{Introduction}

The Glasgow neck lump clinic database is the largest known case series of its type, operating an accepted alternative (of near-site radiological-guided biopsy) to the 'gold standard' model suggested in 2004 by the National Institute for Health and Care Excellence. We asked ourselves, what evidence is there to support an ongoing need for neck lump clinics?

\section{Methods and materials}

Prospective data were collected from October 2005 to January 2016. Data collected included patient demographics, examination findings, diagnosis, and radiological and pathological findings. These were recorded onto a database during clinic and statistically analysed using the Excel ${ }^{\circledR}$ spreadsheet package.

\section{Results}

A total of 5042 patients were seen. Of these patients, 84.5 per cent were referred by the general practitioner. This one-stop first referral clinic assessed both routine and urgent cases, with mean referral-to-clinic time of 26 days. A huge variety of conditions were encountered, but over 50 per cent of patients were definitively managed with just one out-patient appointment. In addition, we found that clinical examination alone was not sufficiently accurate. In our representative sample, one-third of the patients with clinically diagnosed salivary tumours were over-diagnosed.

\section{Conclusion}

Our findings confirm that neck lump clinics serve as a useful one-stop clinic. They help to improve diagnostic speed and reliability, and relieve resource and cost pressures on the National Health Service. In addition, they help prevent undue stress, anxiety and loss of earnings associated with multiple attendances. Clinics can be tailored to suit the local expertise, so long as this is an addition to clinical examination.
Mind over matter: scar satisfaction and body image in thyroidectomy patients - a prospective study

P Sethukumar, D Ly, Z Awad, N Tolley

St Mary's Hospital, Paddington, UK

\section{Introduction}

A study was conducted to determine the impact of body image on scar satisfaction in patients undergoing thyroidectomy in a tertiary referral centre, using validated assessment tools. The impact of life quality, scar size, scar age and patient age were also investigated.

\section{Methods and materials}

A total of 123 patients were included. Patients and clinicians completed the following: the Manchester Scar Scale, to assess scar satisfaction; the Dysmorphic Concern Questionnaire, to assess body image; the Body Dysmorphic Concern Questionnaire, to screen for body dysmorphic disorder; and the EQ$5 \mathrm{D}^{\mathrm{TM}}$, to assess life quality. Patients underwent standardised photography of thyroidectomy scars. An image assessment panel, comprising experts and non-experts, rated scars using the Manchester Scar Scale.

Results

Poor body image was associated with poor scar perception $(\rho=0.178, p=0.05)$. The prevalence of body dysmorphic disorder was 8.94 per cent, which is higher than general population rates. Poor life quality was significantly correlated with poor scar perception $(\rho=-0.292, p=0.001)$. Scar length did not affect scar perception. Clinicians significantly underestimated scar severity. High intra-rater reliability was confirmed using the Manchester Scar Scale image assessment tool.

\section{Conclusion}

This study is the first of its kind to assess the impact of body image on thyroidectomy scar cosmesis. Negative body image and life quality adversely affects thyroidectomy scar perception. The Manchester Scar Scale Image Assessment Scale is a reliable tool for assessing scar photography, and is a useful adjunct in the evaluation of scar cosmesis. Further studies are 
required to assess the prognostic bearing of factors such as body image on scar perception.

A case-control study looking at demographic factors associated with non-attendance at ENT day surgery

R Srinivasan, R Fox, A Singh

Northwick Park Hospital, London, UK

\section{Introduction}

Non-attendance at appointments for surgery has important clinical and financial implications. We investigated those patients who did not attend a day surgical unit at our centre in an attempt to identify factors associated with nonattendance.

\section{Methods and materials}

A retrospective case-control study was conducted. Fortynine consecutive patients who did not attend their ENT day-case operation from July 2014 to February 2016 were identified; these individuals were matched with a control group of 49 consecutive patients who attended their operation. Demographic information were collected and used to calculate indices of deprivation that encompassed, health, education, employment and an overall 'index of multiple deprivation'.

Results

There was a statistically significant $(p<0.01)$ association between the type of surgery planned and non-attendance. Specifically, patients who had nasal procedures planned were more likely to not attend than those scheduled for otological or laryngological procedures. There was no significant association $(p>0.05)$ between age or sex and non-attendance in the two groups. There was also no significant association in terms of specific indices for income, education, health or employment.

\section{Conclusion}

Planned nasal operations were associated with an increased tendency for non-attendance to surgery. These data could be used to guide patient selection for surgery, although further research is required to investigate this association.

Prognostic factors in human papilloma virus associated oropharyngeal carcinoma

M J Ward, T Mellows, E L Kershaw, C Riley, E V King, G J Thomas

University of Southampton School of Medicine, UK

\section{Introduction}

Survival in human papilloma virus (HPV) positive oropharyngeal squamous cell carcinoma (SCC) is better than in HPV-negative oropharyngeal SCC, regardless of the treatment provided. This could lead to less intensive treatment required for some patients, with the same optimal outcome and reduced exposure to side effects. However, a small group of HPV-positive patients have poor outcomes and they currently cannot be identified. This is impeding the use of trials to assess the use of less intensive treatment modalities.

\section{Methods and materials}

A retrospective study of 285 patients was conducted, investigating demographics, prognostic factors, treatment modalities, HPV status and tumour-infiltrating lymphocytes (using immunohistochemistry to accurately categorise the immune response).

\section{Results}

Fifty-four per cent of patients had HPV-positive tumours, with an improved survival outcome compared to their HPV-negative counterparts. The tumour-infiltrating lymphocyte levels were higher in HPV-positive patients, and patients with higher tumour-infiltrating lymphocyte levels had better outcomes. The HPV-positive patients with low tumour-infiltrating lymphocyte levels had similar survival outcomes to HPV-negative patients.

\section{Conclusion}

The HPV-positive patients with low tissue immune infiltrates are at as high risk as HPV-negative patients. 